\title{
The Relationship Between Vertebral Artery Hypoplasia and Posterior Circulation Stroke
} \\ Yüksel Erdal1, (D Banu Bayramoğlu², (D Çağla Şișman¹, (D) Ahmet Batuhan Demiral1, \\ (D) Abdullah Soydan Mahmutoğlu³, (D) Ufuk Emre1 \\ 1University of Health Sciences Turkey, İstanbul Training and Research Hospital, Clinic of Neurology, istanbul, Turkey \\ 2University of Health Sciences Turkey, Bakirköy Prof. Dr. Mazhar Osman Training and Research Hospital for Psychiatric, Neurologic, and Neurosurgical \\ Diseases, Clinic of Neurology, İstanbul, Turkey \\ 3University of Health Sciences Turkey, İstanbul Training and Research Hospital, Clinic of Radiology, İstanbul, Turkey
}

\section{ABSTRACT}

Introduction: Vertebral artery hypoplasia (VAH) is a common variation; however, its role in posterior circulation stroke (PCS) has not been fully elucidated. Thus, this study aimed to evaluate the relationship between VAH and PCS with clinical and laboratory parameters.

Methods: Between January 2016 and June 2020, 178 patients with PCS who were hospitalized in the neurology department were included. The demographic characteristics, vascular risk factors, stroke patterns, and the National Institutes of Health Stroke Scale (NIHSS) and modified Rankin Scale (mRS) scores of patients were recorded. Patients with VA diameter of $\leq 2.0 \mathrm{~mm}$ or 1:1.7 ratio difference in computed tomography angiography were included in the VAH group.

Results: This study included 115 females and 63 males. The mean age of patients was $65.8 \pm 12$ years. VAH was determined in 74 (41.6\%) patients, whereas none in 104 patients (58.4\%). No significant difference was determined in terms of gender and age in patients with and without VAH ( $p=0.310$ and $p=0.676$, respectively). No statistically significant difference was found between the two groups in terms of vascular risk factors $(p>0.05)$. Lacunar stroke pattern was less frequently found in patients with VAH ( $p=0.045)$. Other stroke patterns were similar in both groups $(p>0.05)$. The NIHSS $(p=0.01)$ and $\mathrm{mRS}(p=0.018)$ scores were significantly higher in patients with $\mathrm{VAH}$ than those without.

Conclusion: The presence of VAH in PCS may adversely affect the clinical severity.

Keywords: Vertebral artery hypoplasia, posterior circulation stroke, clinical severity, stroke pattern

\section{Introduction}

Posterior circulation stroke (PCS) occurs within the vascular region of the vertebrobasilar arterial system. Anatomical variations are very common in the posterior circulation vessels, and the vertebral arteries, particularly, are highly variable in diameter, length, and course $(1,2)$. Vertebral artery hypoplasia (VAH) is a common embryonic variation of the posterior circulation (mostly seen in the right) with reported frequencies between $1.9 \%$ and $26.5 \%$. Despite its high prevalence, relatively little information is known about the clinical significance of VAH (3).

$\mathrm{VAH}$ has become the focus of interest in many studies that showed it as a predisposing factor for stroke, especially when accompanied by atherosclerotic risk factors $(4,5)$. However, some uncertainties are still presented. First of all, VAH has no standard definition; currently, VA diameters ranging from 2 to 3 $\mathrm{mm}$ or asymmetry ratios of $\geq 1: 1.7$ have been identified in different studies. Moreover, the role of $\mathrm{VAH}$ as a stroke risk factor remains controversial, while its etiology and pathogenesis are unclear (6).
PCS may present with a wide range of clinical signs due to the alterations in the vertebrobasilar system and the clinical severity of the stroke itself. This situation makes the diagnosis difficult for vascular neurologists $(1,6)$. Studies that suggested an association between VAH and PCS are mainly prevalence-based and provide data in the context of relationships between risk factors and PCS (7-11). A limited number of studies demonstrated the clinical severity of VAH and PCS (12-14). Therefore, our study aimed to evaluate the relationship between VAH and PCS and determine the extent of their clinical association.

\section{Methods}

\section{Patients}

We retrospectively screened all patients with an acute ischemic stroke who are admitted to our clinic between January 2016 and June 2019. Among the patients with a definite acute ischemic stroke diagnosis with diffusion-weighted imaging, patients with PCS and computed 
tomography angiography (CTA) taken within 1 week of admission were included in the study. Patients with bilateral VAH, vertebral artery dissection, total occlusion, and hemorrhagic infarction and patients with lacking information about the initial National Institutes of Health Stroke Scale (NIHSS) and modified Rankin Scale (mRS) score were excluded from the study, thus a final total of 178 patients were included. The study was approved by the University of Health Sciences Turkey, İstanbul Training and Research Hospital Local Ethics Committee (approval number: 1855, 24.05.2019).

The demographic characteristics and vascular risk factors [diabetes mellitus (DM), cardiac disease, hyperlipidemia, smoking, and arterial hypertension] of patients were recorded. The ischemic lesion patterns were radiologically grouped as territorial, embolic $>1.5 \mathrm{~cm}$ in diameter or a single lesion that affects a vascular site with multiple acute lesions), and lacuna (isolated lesion with a diameter of $\leq 1.5 \mathrm{~cm}$ ). Additionally, posterior circulation ischemic lesions were divided into the following five groups based on anatomy: bulbus, pons, mesencephalon, cerebellum, and occipital (Table 1).

\section{Clinical Assessment}

Stroke severity was measured by the NIHSS score (range: 0-42) and mRS-a neurological functional disability scale-ranging from 0 to 6 at 90 days after stroke.

\section{Vessel Analysis}

A Toshiba Aquilium 64 CT Scanner (Toshiba Medical Systems Corporation, Shimoishigami, Japan) was used for all head and neck CTA scans (120 kV, $494 \mathrm{~mA}, 0.5 \mathrm{~mm}$ thickness, $40 \mathrm{~cm}$ FOV, $25.9 \mathrm{~cm}$ reconstruction diameter, $512 \times 512$ matrix, and $500 \mathrm{~ms}$ exposure time). Bolus triggering was used for optimum enhancement with the administration of $350 \mathrm{mgl} / \mathrm{mL}$ contrast media at a rate of $4-5 \mathrm{~mL} / \mathrm{s}$. Multiplanar reformations, threedimensional volume renderings, maximum-intensity projections, and curved planar reformations were post-processed from the source images obtained from the aortic arch to the vertex.

The CTA images of all patients were evaluated based on the VAs. VAH was defined as follows: diameter of $\leq 2.0 \mathrm{~mm}$ or a difference in diameter with the contralateral side that is $>1: 1.7(3,10,11)$.

\section{Statistical Analysis}

For descriptive statistics, mean, standard deviation, median, minimum, and maximum values were used for quantitative variables, and frequency and percentage values were used for qualitative variables. The distribution of variables was measured using the KolmogorovSmirnov test. Independent samples t-test and the Mann-Whitney $U$ test were used depending on distribution normality for the analysis of quantitative independent data. The chi-square test was used for the analysis of qualitative independent data and the Fisher's test when data did not meet the chi-square assumptions. All statistical analyses were performed using the Statistical Package for the Social Sciences version 22.0 software (SPSS Inc., Chicago, IL, USA). A p-value of $\leq 0.05$ was considered significant.

\section{Results}

Of the 178 patients with acute PCS, 115 (64.6\%) were female and 74 (41.6\%) were diagnosed with VAH. No significant difference was found in gender (female/male: $51 / 23$ vs $64 / 40, p=0.310$ ) and age ( $65.4 \pm 10.6$ vs $66.1 \pm 12.9, p=0.676)$ distribution of the groups with and without $\mathrm{VAH}$. Among the patients with VAH, 44 (59.5\%) had right-sided.

No statistically significant difference was found between the two groups in terms of vascular risk factors (hypertension, DM, hyperlipidemia, coronary artery disease, and smoking) ( $p>0.05)$.

The NIHSS score was $3.8 \pm 2.9$ and mRS was $1.6 \pm 1.1$ in patients with VAH, whereas $2.4 \pm 1.7$ and $1.2 \pm 0.9$ in the patients without $\mathrm{VAH}$, respectively. The NIHSS $(p=0.01)$ and $m R S(p=0.018)$ scores were significantly higher in patients with VAH compared to patients without VAH.

In terms of the stroke patterns, the lacunar stroke pattern was significantly more common in patients without VAH $(p=0.045)$, while other stroke patterns were similar in both groups $(p>0.05)$. Posterior circulation infarct localization did not significantly differ in the patients with and without VAH ( $p>0.05)$ (Table 2).

\begin{tabular}{|c|c|c|c|}
\hline & & $\begin{array}{l}\text { Minimum- } \\
\text { maximum }\end{array}$ & Mean \pm SD $/(n, \%)$ \\
\hline \multicolumn{2}{|l|}{ Age } & $28.0-89.0$ & $65.8 \pm 12.0$ \\
\hline \multirow{2}{*}{ Gender } & Female & - & $115(64.6 \%)$ \\
\hline & Male & - & $63(35.4 \%)$ \\
\hline \multirow{2}{*}{ VAH } & $(+)$ & - & $74(41.6 \%)$ \\
\hline & $(-)$ & - & $104(58.4 \%)$ \\
\hline Right VAH & & - & $44(59.5 \%)$ \\
\hline Left VAH & & - & $30(40.5 \%)$ \\
\hline \multicolumn{4}{|l|}{ Clinical assessment } \\
\hline NIHSS & & $0.0-15.0$ & $3.0 \pm 2.5$ \\
\hline $\mathrm{mRS}$ & & $0.0-5.0$ & $1.4 \pm 1.0$ \\
\hline \multicolumn{4}{|l|}{ Risk factors } \\
\hline Hyperlipidemia & & - & $116(65.2 \%)$ \\
\hline Diabetes & & - & $73(41.0 \%)$ \\
\hline Arterial hypertension & & - & $121(68.0 \%)$ \\
\hline Cigarette smoke & & - & $52(29.2 \%)$ \\
\hline Coronary heart disease & & - & $38(21.3 \%)$ \\
\hline \multicolumn{4}{|l|}{ Stroke pattern } \\
\hline Territorial & & - & $82(46.0 \%)$ \\
\hline Lacunar & & - & $53(29.8 \%)$ \\
\hline Embolic & & - & $43(24.2 \%)$ \\
\hline \multicolumn{4}{|l|}{ Location of infarction } \\
\hline Medulla oblongata & & - & $23(12.9 \%)$ \\
\hline Pons & & - & $74(41.6 \%)$ \\
\hline Mesencephalon & & - & $8(4.5 \%)$ \\
\hline Cerebellum & & - & $56(31.5 \%)$ \\
\hline Occipital lobe and thalamus & & - & $28(15.7 \%)$ \\
\hline
\end{tabular}

SD: Standard deviation, VAH: vertebral artery hypoplasia, NIHSS: National Institutes of Health Stroke Scale, mRS: modified Rankin Scale 


\begin{tabular}{|c|c|c|c|c|c|}
\hline \multirow{2}{*}{\multicolumn{2}{|c|}{ Age }} & \multirow{4}{*}{$\begin{array}{l}\text { VA hypoplasia } \\
(+)(\mathbf{n}=74) \\
65.4 \pm 10.6 \\
51(68.9 \%) \\
23(31.1 \%)\end{array}$} & \multirow{4}{*}{$\begin{array}{l}\text { VA hypoplasia } \\
(-)(n=104) \\
66.1 \pm 12.9 \\
64(61.5 \%) \\
40(38.5 \%)\end{array}$} & \multicolumn{2}{|l|}{ p } \\
\hline & & & & 0.676 & $\mathrm{~m}$ \\
\hline \multirow{2}{*}{ Gender } & Female & & & \multirow{2}{*}{0.310} & \multirow{2}{*}{$X^{2}$} \\
\hline & Male & & & & \\
\hline \multicolumn{6}{|l|}{ Risk factors } \\
\hline \multicolumn{2}{|l|}{ Hyperlipidemia } & $48(64.9 \%)$ & $68(65.4 \%)$ & 0.943 & $X^{2}$ \\
\hline \multicolumn{2}{|l|}{ Diabetes } & $26(35.1 \%)$ & 47 (45.2\%) & 0.179 & $X^{2}$ \\
\hline \multicolumn{2}{|c|}{ Arterial hypertension } & $48(64.9 \%)$ & $73(70.2 \%)$ & 0.453 & $x^{2}$ \\
\hline \multicolumn{2}{|l|}{ Cigarette smoke } & $23(31.1 \%)$ & $29(27.9 \%)$ & 0.644 & $X^{2}$ \\
\hline \multicolumn{2}{|c|}{ Coronary heart disease } & $16(21.6 \%)$ & $22(21.2 \%)$ & 0.940 & $x^{2}$ \\
\hline \multicolumn{6}{|c|}{ Clinical assessment } \\
\hline \multicolumn{2}{|l|}{ NIHSS score } & $3.8 \pm 2.9$ & $2.4 \pm 1.7$ & 0.010 & $\mathrm{~m}$ \\
\hline \multicolumn{2}{|l|}{ mRS score } & $1.6 \pm 1.1$ & $1.2 \pm 0.9$ & 0.018 & $\mathrm{~m}$ \\
\hline \multicolumn{6}{|l|}{ Stroke pattern } \\
\hline \multicolumn{2}{|l|}{ Territorial } & $37(48.6 \%)$ & $45(44.1 \%)$ & 0.310 & $X^{2}$ \\
\hline \multicolumn{2}{|l|}{ Lacunar } & $16(21.1 \%)$ & 37 (36.3\%) & 0.045 & $X^{2}$ \\
\hline \multicolumn{2}{|l|}{ Embolic } & $23(30.3 \%)$ & $20(19.6 \%)$ & 0.069 & $x^{2}$ \\
\hline \multicolumn{6}{|c|}{ Location of infarction } \\
\hline \multicolumn{2}{|c|}{ Medulla oblongata } & $11(14.9 \%)$ & $12(11.5 \%)$ & 0.514 & $x^{2}$ \\
\hline \multicolumn{2}{|l|}{ Pons } & $32(43.2 \%)$ & $42(40.4 \%)$ & 0.703 & $x^{2}$ \\
\hline \multicolumn{2}{|l|}{ Mesencephalon } & $2(2.7 \%)$ & $6(5.8 \%)$ & 0.330 & $x^{2}$ \\
\hline \multicolumn{2}{|l|}{ Cerebellum } & $27(36.5 \%)$ & $29(27.9 \%)$ & 0.240 & $X^{2}$ \\
\hline \multicolumn{2}{|l|}{$\begin{array}{l}\text { Occipital lobe and } \\
\text { thalamus }\end{array}$} & $8(10.8 \%)$ & $20(19.2 \%)$ & 0.128 & $X^{2}$ \\
\hline
\end{tabular}

m: Mann-Whitney U test, ${ }^{{ }^{2}}$ : chi-square test (Fischer's test), VAH: vertebral artery hypoplasia, Boldface values indicate significant results $(p<0.05)$.

\section{Discussion}

This study assessed the association between VAH and PCS by retrospective analysis of patient data and revealed some similarities and differences from studies on this subject in the last 20 years, which seems to be a trend in studies of this type. However, the most important output of our study was the worse clinical severity in patients who suffered from posterior ischemic stroke in the presence of $\mathrm{VAH}$. Despite the limitations that are present in this study and many studies in this field (due to the inability to perform population-based analyses), this is an important finding that could help in clinical settings.

$\mathrm{VAH}$ is common in society; however, there are differences between the prevalence values. The incomplete consensus on VAH definition and the use of different measurement methods [magnetic resonance angiography (MRA), CTA, and ultrasonography] in studies may explain the reason for these differences. A review by Katsanos and Giannopoulos (6) revealed that VAH was two times more common in PCS than in anterior system stroke. A study with 129 patients with acute stroke by Mitsumura et al. (8) identified $44.4 \%$ of VAH cases in patients with PCS. Similarly, among the 750 patients with stroke evaluated by Kulyk et al. (13), 193 have PCS and 33.7\% of this group had VAH. Another similar percentage comes from a study by Park et al. (11), who revealed that VAH was present in $45.6 \%$ of patients with PCS. Our study revealed that $41.6 \%$ of patients had VAH. We could not perform direct comparisons with these previous studies because anterior system strokes were excluded from our study. However, our findings, at least, seem to be consistent with previous studies in terms of the relationship between PCS and VAH.

Previous studies have predominantly demonstrated the relationship between prevalence and risk factors (7-11). VAH has been studied in a limited number of studies in terms of clinical outcome, and these have not proposed any association with VAH and stroke severity (1214). Kulyk et al. (13) revealed that mRS was similar in patients with and without VAH. Yang et al. (14) compared the NIHSS scores in patients with PCS (with and without VAH) and revealed no relationship. A study with 815 patients that had had an acute ischemic stroke, by Sauer et al. (12), revealed no significant difference between the median NIHSS and mRS scores in patients with and without VAH. However, when we look at the details of this study, comparisons were performed in the whole patient group (anterior and posterior) rather than separately (12). Our study revealed that the NIHSS score was $3.8 \pm 2.9$ and the mRS score was $1.6 \pm 1.1$ in patients with $\mathrm{VAH}$. Both scores were significantly higher compared to those without $\mathrm{VAH}$. These findings may provide a new perspective for the clinical severity of PCS with different clinical findings.

Considering the imaging-based studies that examine the relationship between PCS and VAH, Doppler ultrasonography $(7,13,14)$ and MRA $(5,9,13,14)$ are revealed as the most popular methods used. Doppler ultrasonography is a rapid and noninvasive technique for the assessment of vertebral arteries, but it may fail to identify aplasia, hypoplasia, occlusion, or dissection (15). CTA has been shown to have higher sensitivity and positive predictive value in detecting intracranial vessel stenosis and occlusion compared to MRA (16). Our study was performed with the CTA measurement method and we think that it gave more objective results in this sense compared to the other studies.

Different results have been obtained regarding the embolic stroke patterns in patients with VAH. Perren et al. (7) revealed that the embolic stroke pattern was more frequent in patients with VAH. Quite conversely, Sauer et al. (12) revealed that the embolic stroke pattern was less frequent in patients with $\mathrm{VAH}$. Data is limited on this topic, as previously mentioned, and even available data seems to be biased; however, most were probably based on the clinical characteristics of patients, imaging modalities, and genetic/phenotypic differences. Our study revealed that the lacunar stroke pattern was less frequent in patients with $\mathrm{VAH}$, and no differences were found regarding other stroke patterns. This situation can be explained by the possibility that risk factors contributing to the etiopathogenesis of lacunar stroke were more common among patients without $\mathrm{VAH}$.

Many imaging studies have demonstrated that VAH may independently predispose patients to regional hypoperfusion in the posterior circulation (17). Other evidence from relevant research revealed that VAH may lead to ischemic events in the posterior circulation, especially when accompanied by other atherosclerotic risk factors $(4,16,18)$. In our study, the evaluation of risk factors regarding the relationships revealed that none of the vascular risk factors were associated with a significant difference among the groups. 


\section{Study Limitations}

Our study has some limitations. The number of patients was low to draw a generalized conclusion. However, there is very little conclusive data on this topic and available studies have not focused on the relationships between VAH and only PCS. Anterior group strokes were not included in the study by design, thus no comparison could be made concerning VAH frequencies. In a study, a 42\% decrease of flow was detected in the posterior inferior cerebellar artery (PICA) territorial area of the ipsilateral VAH (3). Case reports and some studies revealed a relationship between VAH and ipsilateral PCS, especially PICA strokes (11). However, we did not evaluate the side of hypoplasia and the side of stroke. Additionally, long-term outcomes should be observed to better assess the clinical outcome and severity.

\section{Conclusion}

Our study results suggest a significant relationship between $\mathrm{VAH}$ and clinical severity of stroke in patients with PCS. Detailed prospective and clinical studies with larger groups will be useful to determine the relationship between $\mathrm{VAH}$ and clinical outcomes.

Ethics Committee Approval: The study was approved by the University of Health Sciences Turkey, İstanbul Training and Research Hospital Local Ethics Committee (approval number: 1855, 24.05.2019).

Informed Consent: Retrospective study.

Peer-review: Externally peer-reviewed.

Authorship Contributions: Surgical and Medical Practices - Y.E.; Concept - U.E.; Design - Y.E.; Data Collection or Processing - Y.E., B.B., C.S., A.B.D.; Analysis or Interpretation - U.E., A.S.M.; Literature Search Y.E.; Writing - Y.E., A.S.M., U.E.

Conflict of Interest: No conflict of interest was declared by the authors.

Financial Disclosure: The authors declared that this study received no financial support.

\section{References}

1. Nouh A, Remke J, Ruland S. Ischemic posterior circulation stroke: a review of anatomy, clinical presentations, diagnosis, and current management. Front Neurol 2014; 5: 30.

2. Kim C, Sohn JH, Choi HC. Are the anomalous vertebral arteries more hypoplastic?: retrospective linear mixed model approach. BMC Neurol 2017; 17: 168.

3. Thierfelder KM, Baumann AB, Sommer W, Armbruster M, Opherk C, Janssen $\mathrm{H}$, et al. Vertebral artery hypoplasia: frequency and effect on cerebellar blood flow characteristics. Stroke 2014; 45: 1363-8.
4. Katsanos AH, Kosmidou M, Kyritsis AP, Giannopoulos S. Is vertebral artery hypoplasia a predisposing factor for posterior circulation cerebral ischemic events? A comprehensive review. Eur Neurol 2013; 70: 78-83.

5. Chuang YM, Huang YC, Hu HH, Yang CY. Toward a further elucidation: role of vertebral artery hypoplasia in acute ischemic stroke. Eur Neurol 2006; 55 : 193-7.

6. Katsanos AH, Giannopoulos S. Increased risk for posterior circulation ischaemia in patients with vertebral artery hypoplasia: a systematic review and meta analysis. Eur Stroke J 2017; 2: 171-7.

7. Perren F, Poglia D, Landis T, Sztajzel R. Vertebral artery hypoplasia: a predisposing factor for posterior circulation stroke. Neurology 2007; 68: 65-7.

8. Mitsumura H, Miyagawa S, Komatsu T, Hirai T, Kono Y, Iguchi Y. Relationship between vertebral artery hypoplasia and posterior circulation ischemia. J Stroke Cerebrovasc Dis 2016; 25: 266-9.

9. Gaigalaite V, Vilimas A, Ozeraitiene V, Dementaviciene J, Janilionis R, Kalibatiene D, et al. Association between vertebral artery hypoplasia and posterior circulation stroke. BMC Neurol 2016; 16: 118.

10. Hu XY, Li ZX, Liu HQ, Zhang M, Wei ML, Fang S, et al. Relationship between vertebral artery hypoplasia and posterior circulation stroke in Chinese patients. Neuroradiology 2013; 55: 291-5.

11. Park JH, Kim JM, Roh JK. Hypoplastic vertebral artery: frequency and associations with ischemic stroke territory. J Neurol Neurosurg Psychiatry 2007; 78: 954-8.

12. Sauer T, Wolf ME, Ebert AD, Szabo K, Chatzikonstantinou A. Vertebral artery hypoplasia does not influence lesion size and clinical severity in acute ischemic stroke. J Stroke Cerebrovasc Dis 2016; 25: 1770-5.

13. Kulyk C, Voltan C, Simonetto M, Palmieri A, Farina F, Vodret F, et al. Vertebral artery hypoplasia: an innocent lamb or a disguise? J Neurol. 2018; 265: 234652.

14. Yang J, Shen Z, Wen H, Zhou H, Li C. The effect of vertebral artery hypoplasia in posterior circulation infarction in young patients. Int J Neurosci 2016; 126 : 1092-6.

15. Buckenham TM, Wright IA. Ultrasound of the extra-cranial vertebral artery. $\mathrm{Br}$ J Radiol 2004; 77: 15-20.

16. Bash S, Villablanca JP, Jahan R, Duckwiler G, Tillis M, Kidwell C, et al. Intracranial vascular stenosis and occlusive disease: evaluation with $\mathrm{CT}$ angiography, MR angiography, and digital subtraction angiography. AJNR Am J Neuroradiol 2005; 26: 1012-21.

17. Szarazova AS, Bartels E, Bartels S, Turcani P. Possible morphological pathomechanisms of ischemic stroke in the posterior circulation of patients with vertebral artery hypoplasia. J Neuroimaging 2015; 25: 408-14.

18. Giannopoulos S, Markoula S, Kosmidou M, Pelidou SH, Kyritsis AP. Lateral medullary ischemic events in young adults with hypoplastic vertebral artery. J Neurol Neurosurg Psychiatry 2007; 78: 987-9. 